organisation that can be illuminated by evolutionary thinking. A crisp review of some of this work is due to Kolata (Science, 187, 50-51 and 156$157 ; 1975)$. When one comes to the primates (excluding man), social structures become very complicated and diverse, ranging from troops with a complex social organisation (baboons), through nuclear families (white-handed gibbons, with a single mated pair accompanied by up to four immature offspring and an occasional aged male), to individuals who spend most of their lives in solitary fashion (male orangutans).

So far I have touched on 25 of the 27 chapters in Sociobiology, which deal with non-human animals. This work constitutes a tour de force of synoptic scholarship, and has not been challenged.

\section{Human behaviour}

In chapters 1 and 27, Wilson speculates upon the insights to be gained by applying sociobiological thinking to human behaviour and human societies.
The social insects, for example, show how extreme forms of altruism can evolve by virtue of kin selection in conjunction with inbreeding. In the absence of any constraints, there is a strong selective advantage in inbreeding coupled with recognition and cooperation among kin. But in relatively complex organisms, such as humans, there is a severe constraint, namely the well documented increase in the expression of deleterious genetic traits as heterozygosity is diminished by inbreeding. Wilson poses the question: how are the gains through inclusive fitness and losses through inbreeding depression balanced? In which life history strategies is this balance struck so as to increase the likelihood of more intense inbreeding and social life? In particular, he observes that in human societies (excepting Faulkner's Yoknapatawpha County) the incest taboo is both universal and has the merit of minimising the occurrence of crippling genetic malformations. $\mathrm{He}$ suggests that this universal taboo has adaptive origins, rooted in some gene complex.
In suggesting that pervasive human behavioural manifestations such as spite, indoctrinability or courage may have aspects that are genetically controlled, Wilson is at pains to emphasise that he is not implying "that there exists one gene for spite, another for homosexuality, and so on, as one might envisage the inheritance of flower colour or seed texture in garden peas. The tendency to develop such behaviour, in a distinctively human form, is part of an immensely complex social repertory which is undoubtedly dependent on large complexes of genes" (Harvard Gazette, January 16, 1976). Given that the relatively simple and stereotyped behaviour of aggregation in slime moulds is under the control of a complex of about 50 genes (Cox, Princeton University, personal communication), I find Wilson's view plausible. It is, however, clear that direct confirmation, in the form of explicitly identifying particular gene complexes for particular behaviour, is out of the question.

These first and last chapters, with their suggestion that human behaviour
ONE long-standing objective in polymer science is the production of fibres with an elastic modulus (ratio of stress to strain) approaching the theoretical maximum value. It seems self-evident that the structure should consist of polymer chains aligned as closely as possible to the fibre axis with few folds or disoriented regions. Various methods might produce this structure. The natural and most elegant method is exemplified in the production of plant cellulose where polymerisation occurs in situ so that fibres grow by addition of monomer units to one end. This has never really been achieved synthetically.

The drawing process used in the production of textile fibres produces chain orientation by stretching the fibre to several times its initial length. Two fibre industry alumni now in universities have worked on modifying this process to produce high draw ratios and orientation and so high moduli. E. S. Clark of the University of Tennessee and co-workers at Du Pont have produced acetal fibres with a modulus of $35 \mathrm{GPa}$ (1 giga pascal= $10^{9} \mathrm{~N} \mathrm{~m}^{-3}$ ) (Polymer Engng Sci., 14, 682 ; 1974). For comparison, carbon fibre has a modulus of $500 \mathrm{GPa}$ and the theoretical maximum modulus of polyethylene is $220 \mathrm{GPa}$ (Frank, Proc. $R$. Soc. Lond., A319, 127; 1970). These acetal fibres were drawn at about $130^{\circ} \mathrm{C}$ by a factor of 20 times in a two-stage process. The usual room temperature draw ratio is 7 times. The structure seems to consist of a single highly oriented phase.
I. M. Ward and co-workers at Leeds have studied the dependence of draw ratio on temperature, molecular weight and pre-draw microstructure in polyethylene. They found that the modulus increases roughly linearly to $70 \mathrm{GPa}$ at a draw ratio of 30 times. The draw ratio was a maximum for a sample of high molecular weight and narrow molecular weight distribution crystallised by cooling to $110^{\circ} \mathrm{C}$ then quenching to room

\section{Strong fibres}

from Paul Calvert

temperature and drawn at $75^{\circ} \mathrm{C}$ (Polymer, 15, 233; 1974: Nature phys. Sci., 243, 143; 1973). This leaves the question as to whether it is the molecular weight itself or its influence on the microstructure that is important in allowing a high draw ratio.

Barham and Keller (J. Materials Sci., 11, 27; 1976) have taken up this point and looked at the relationship between modulus, draw ratio and starting structure. They find that the dependence of modulus on draw ratio is the same for a range of pre-draw microstructures. What did change with microstructure was the maximum draw ratio which could be attained. Sheet which had been directly quenched or made from a sample with no low molecular weight polymer could not be drawn past 20 25 times as opposed to $30-35$ times. This point is somewhat confused in the paper by the fact that the figure captions are exchanged.

From this Barham and Keller deduce that the low molecular weight chains, which segregate to the spherulite boundaries during slow crystallisation, govern the drawability of the sample. In support of this they say that samples which draw well had a black line around the spherulites when viewed with crossed polars. Presumably this region is liquid at the drawing temperatures and somehow allows large deformations without fracture. This is surprising in that one would expect strong rather than weak boundaries to be necessary.

Finally, mention should be made of the high melting point, high modulus fibres produced by spinning aromatic polyamides and polyhydrazides from strongly hydrogen bonding solvents such as dimethylacetamide-lithium chloride or sulphuric acid in which they form lyotropic liquid crystals. These fibres spun from anisotropic solutions have moduli several times those of fibres spun from similar isotropic solutions and reach $140 \mathrm{GPa}$. They are reviewed by Carter and Schenk (Structure and properties of oriented polymers, edit. by M. Ward), where the industrial significance is evidenced by the fact that most of the important references are to the patent literature. Two fibres, called Kevlar and Kevlar 49 by Dupont, which are based on poly ( $\mathrm{p}$-phenylene terephthalamide) look likely to be used in tyre belting and in composites for body armour and aircraft parts. 